\title{
Effects of Preparation Conditions on the Yield and Embedding Ratio of Vinyl Silicone Oil Microcapsules
}

\author{
Aijie MA ${ }^{1 *}$, Qiuyu ZHANG ${ }^{2}$, Chunmei LI $^{2}$, Weixing CHEN ${ }^{1}$, Chunyan LUO $^{1}$ \\ ${ }^{1}$ School of Materials and Chemical Engineering, Xi'an Technological University, Xi'an, 710021, P. R. China \\ ${ }^{2}$ Key Laboratory of Applied Physics and Chemistry in Space of Ministry of Education, School of Science, Northwestern \\ Polytechnical University, Xi'an, 710072, P. R. China \\ cross $^{\text {ref }}$ http://dx.doi.org/10.5755/j01.ms.22.2.13026
}

Received 02 September 2015; accepted 19 February 2016

\begin{abstract}
Self-healing materials could repair themselves without external influences when they are damaged. In this paper, microcapsules are prepared by in-situ polymerization method, utilizing vinyl silicone oil as core material, polyurea formaldehyde as wall material and polyvinyl alcohol as dispersant. The morphology and structure of the microcapsules are tested with scanning electron microscopy, optical microscopy and laser particle analyzer. Effect of the reaction temperature, stirring speed and polyvinyl alcohol concentration on the yield, embedding ratio, particle size and its distribution are studied. Results show that the microcapsules can be successfully prepared by in-situ polymerization method. Under the reaction condition of temperature $60{ }^{\circ} \mathrm{C}$, stirring speed $1000 \mathrm{r} / \mathrm{min}$, dispersant concentration $0.1 \mathrm{wt} . \%$, the yield and embedding ratio of the microcapsule are found to be $52.5 \%$ and $50.1 \%$, respectively. The prepared microcapsules have smooth surface, good dispersibility, narrow particle size distribution and the average particle size is $13 \mu \mathrm{m}$.

Keywords: microcapsule, in-situ polymerization, yield, embedding ratio.
\end{abstract}

\section{INTRODUCTION}

Microcapsules are often used as a tiny container to store and rapidly release the core material for self-healing [1]. In recent years, the microcapsules with unique properties are widely used in medicine, pesticides, cosmetics, coatings, printing ink, additives and other fields [2]. The properties of the core and wall material should be both considered when preparing the microcapsules because they are directly related to the stability, permeability, viscosity, mechanical strength, solubility and film-forming properties [3]. Due to the unique structure and properties, microcapsule techniques have been applied in self-healing material production.

The intelligent material refers to those who can imitate living system, have self-diagnosing and self-repairing functions when damaged. The repairing additives are microencapsulated and then the microcapsules are embedded into the substrate material as a self-healing function unit. In response to damage, autonomic repair is triggered by rupture of the microcapsules. The repairing agents could flow out of the microcapsules, repair the substrate and prolong the service life of materials. And it can not only improve the comprehensive performance of the material, but also avoid the waste of resources and lower the cost. At present, the microcapsules used in selfhealing applications have gradually got the attention in the military equipment, aerospace, electronics, automobiles, building materials and other fields of science and technology [4-5].

To achieve the release and self-healing function, the composition and structure of microcapsule materials are

\footnotetext{
* Corresponding author. Tel.: +086-29-86173324;

fax: +086-29-86173324. E-mail address: maaijie@xatu.edu.cn (A. Ma)
}

very important. Many preparation methods of microcapsule have developed in recent years. The in-situ polymerization method can effectively be used to control the wall thickness and core content of microcapsule. In particular, the in-situ polymerization involves monomer intercalation followed by polymerization. Instead of a simple dissolution/precipitation physical process, it can facilitate extraordinarily high coverage and shell strength owing to the chemical polymerization occurred on the surface of core material [6]. This method has been used wildly for good healing performance, simple preparation technology and low cost of products [7-8].

For instance, Rule et al. investigated the influence of microcapsule diameter and crack size on the performance of self-healing materials [9]. Results show that the size and weight fraction of microcapsules deliver to a crack face is shown to scale linearly with microcapsule diameter for a given weight fraction of capsules. Keller et al. encapsulated PDMS resin and crosslinker into the poly (dimethyl siloxane) (PDMS) matrix and studied the healing efficiency and mechanism [10]. They found the addition of microcapsules increases the tear strength of the PDMS and self-healed PDMS specimens routinely recover between $70-100 \%$ of the original tear strength. Caruso et al. embed chlorobenzene / urea-formaldehyde microcapsules in the epoxy resin matrix. The release of repair agent chlorobenzene are induced by crack propagation and self-repair function is realized when the matrix is damaged [11]. Fan et al. prepared glass beads/PUF microcapsules by in-situ polymerization [12]. They found that the surface morphology of the microcapsule mainly depends on the $\mathrm{pH}$ value and stirring rate. Smoother microcapsules could be prepared with a higher final $\mathrm{pH}$ value and faster stirring rate. 
As the repair unit, the yield and core content of the microcapsules are particularly important. The self-healing efficiency can obviously increase with the increased content of core material. According to the requirements of the final application, we prepare microcapsules by in-situ polymerization with UV-curable vinyl siliconeoil as core material and PUF as shell material. This paper focuses on the effects of preparation conditions on the yield, embedding ratio and particle size distribution of microcapsules. Finally, the structure and properties of microcapsules are characterized and tested.

\section{EXPERIMENTAL PROCEDURE}

\subsection{Reagents and materials}

Vinyl silicone oil (V401-1000) was purchased from Dayi Chemical Co., Ltd. Formaldehyde, concentrated hydrochloric acid, sodium hydroxide $(\mathrm{NaOH})$, xylene, hydroxyphenol were purchased from Tianli Chemical Reagent Co., Ltd. Polyvinyl alcohol, was purchased from Tianxiang Chemical Industrial Co., Ltd. Urea, nonylphenol polyoxyethylene ether (OP-10), sodium polyacrylic acid, anhydrous ethanol, chemical pure were purchased from Dongfang Chemical Reagent Factory. Ammonium chloride was purchased from the elaboration of Shanghai Chemical Reagent Factory. Gelatin, biochemical reagents were purchased from Tianjin Chemical Reagent Co., Ltd.

\subsection{Preparation of PUF coated vinyl silicone oil microcapsule}

$2.0 \mathrm{~g}$ urea and $0.1 \mathrm{~g}$ hydroxyphenol were dispersed in $100 \mathrm{ml}$ aqueous solution of PVA via stirring with $1000 \mathrm{rpm}$ speed at $80^{\circ} \mathrm{C}$. Then the $\mathrm{pH}$ value was adjusted to 3.0 and a designed amount of vinyl silicone oil was added into the solution. $0.5 \%$ ammonium chloride was then dissolved into the aqueous solution to keep the systems dispersing stably. The reaction temperature was reduced to $60^{\circ} \mathrm{C}$ and then $6.0 \mathrm{~g} 37 \%$ formaldehyde solution was added with continuous stirring for $2 \mathrm{~h}$. Afterwards, the microcapsules were washed separately with xylene and ethanol, filtered and dried at room temperature $\left(25^{\circ} \mathrm{C}\right)$.

\subsection{Testing and characterization}

The particle size and distribution of microcapsules were tested by the laser particle size analyzer (American Beckman Coulter Co., Ltd., USA). The morphology of microcapsules was observed by a $2500 \mathrm{P}$ polarizing microscope (Leica Co., Ltd., Germany) and a $400 \mathrm{~F}$ Field Emission Scanning Electron Microscope. (FEI Co., Ltd., USA).

The microcapsules are extracted by soxhlet's extracter for $24 \mathrm{~h}$ and dried at room temperature for weighing $\left(m_{1}\right)$. Then the core material is extracted with ethyl alcohol as extractor and weighed $\left(m_{2}\right)$. The yield of microcapsule is calculated according to:

yield $=\frac{m_{1}}{m_{0}} \times 100 \%$

where $m_{0}$ is mass of microcapsules (unit: $\mathrm{g}$ ); $m_{1}$ is total mass of all raw materials (unit: $\mathrm{g}$ ).
The embedding ratio of core material is calculated according to:

embedding ratio $=\frac{m_{2}}{m_{3}} \times 100 \%$

where $m_{2}$ is mass of core (unit: $\mathrm{g}$ ); $m_{3}$ is mass of all vinyl silicone oil (unit: g).

\section{RESULTS AND DISCUSSION}

\subsection{Effect of reaction temperature on the yield and embedding ratio of microcapsules}

Some microcapsules are prepared with the stirring speed of $1000 \mathrm{r} / \mathrm{min}$ and the mass percent concentration of $0.1 \%$ PVA aqueous solution at different temperature. Effect of reaction temperature on the yield and embedding ratio of microcapsules is discussed and the result is shown in Table 1 and Fig. 1.

Table 1. Effect of reaction temperature on the yield and embedding ratio of microcapsules

\begin{tabular}{|l|c|c|c|c|}
\hline $\mathrm{T},{ }^{\circ} \mathrm{C}$ & 50 & 60 & 70 & 80 \\
\hline Yield, \% & 38.0 & 48.1 & 40.2 & 25.1 \\
\hline Embedding ratio, \% & 47.8 & 56.2 & 51.5 & 4.6 \\
\hline
\end{tabular}

As can be seen from Table 1, the yield and embedding ratio increase firstly and decrease subsequently with the increase of temperature and then go for the maximum $48.1 \%$ and $56.2 \%$ at $60{ }^{\circ} \mathrm{C}$. With the increase of reaction temperature, precipitation speed of PUF increase, and embedding ratio increase together. When the temperature is higher than $60^{\circ} \mathrm{C}$, the polymerization rate and precipitation speed of PUF increase are too fast to encapsulate the core material gradually. At $80{ }^{\circ} \mathrm{C}$, there is only a lot of solid PUF spheres formed and most vinyl silicone oil droplet suspended in reaction system.

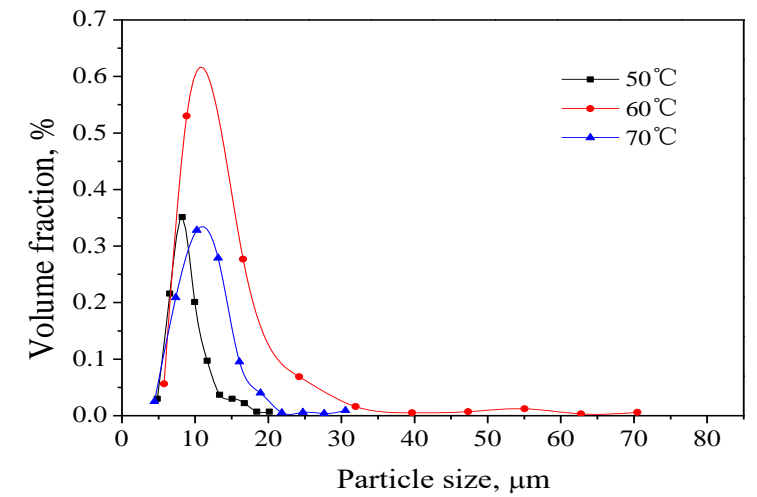

Fig. 1. Effect of temperature on particle size and distribution of microcapsules

We can conclude that the peaks at $10 \mu \mathrm{m}$ and $42 \mu \mathrm{m}$ represent the particle size of vinyl silicone oil droplet and PUF solid spheres respectively. Results show that the optimum temperature is $60^{\circ} \mathrm{C}$, and the yield and embedding ratio of microcapsules prepared at this temperature. The average particle size is about $13 \mu \mathrm{m}$ and particle size distribution is narrow.

\subsection{Effect of stirring rate on the yield and embedding ratio of microcapsules}

PUF microcapsules are prepared at the temperature of $60{ }^{\circ} \mathrm{C}$ and the mass percent concentration of PVA aqueous 
solution $0.1 \%$ at different stirring rate. Effect of stirring rate on the yield and embedding ratio of microcapsules is discussed and the results are shown in Table 2 and Fig. 2.

Table 2. Effect of stirring rate on the yield and embedding ratio of microcapsules

\begin{tabular}{|c|c|c|c|c|c|}
\hline Stirring rate, r/min & 600 & 800 & 1000 & 1200 & 1400 \\
\hline Yield, \% & 32.0 & 48.1 & 52.5 & 29.4 & 28.9 \\
\hline Embedding ratio, wt.\% & 39.8 & 50.5 & 49.1 & 40.8 & 36.3 \\
\hline
\end{tabular}

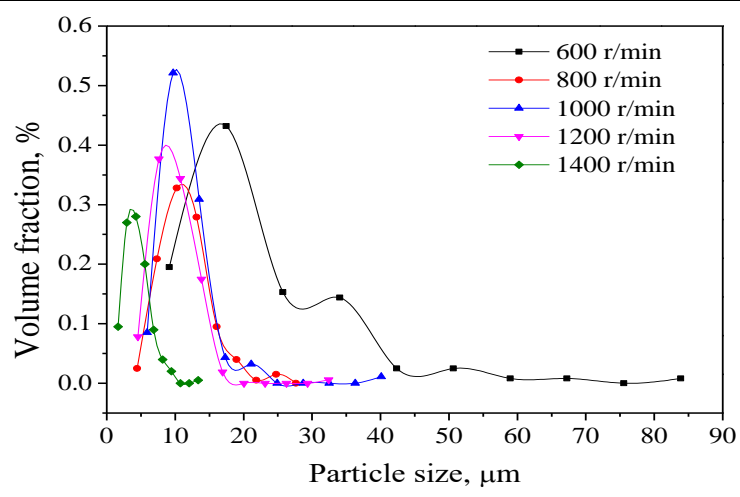

Fig. 2. Effect of stirring rate on the distribution of the microcapsules size

As can be seen from Table 2, the yield and embedding ratio increase firstly and decrease subsequently with the increase of stirring rate. The yield and embedding ratio are $48.1 \%$ and $50.5 \%$ respectively when the stirring rate is $800 \mathrm{r} / \mathrm{min}$. When the stirring speed is increased to $1000 \mathrm{r} / \mathrm{min}$, the yield and embedding ratio are $52.5 \%$ and $49.1 \%$ respectively. Fig. 2 demonstrates that the average diameter and distribution of disperse particles become larger with the decrease of the stirring rate. It is because the reaction emulsion is hard to disperse at low stirring speed. Then the polymerization and precipitation speed of PUF are too slow to encapsulate all vinyl silicone oil. It will lead to low yield and embedding ratio and small particle sizes of microcapsules. With the increase of stirring rate, shear stress of reaction system increases and droplet size of dispersion phase decreases accordingly. The stability of emulsion gets better and it is important to match the precipitation rate of PUF with embedding core material. Then the yield and embedding ratio of microcapsules enhance, and the diameter of particles increase and the size distribution turn wider as time going. But with the stirring rate increasing, the polymerization will react more quickly. The molecular weight of PUF also increase and PUF will precipitate out from the solution quickly. At the same time, too large shear stress will cause the microcapsules break and reduce the yield and the embedding ratio. Based on the yield of microcapsules, $1000 \mathrm{r} / \mathrm{min}$ is chosen as the best stirring speed for preparing microcapsule finally.

Table 3. Effect of PVA concentration on the yield and embedding ratio of microcapsules

\begin{tabular}{|c|c|c|}
\hline PVA concentration, wt. $\%$ & Yield, $\%$ & Embedding ratio, wt $\%$ \\
\hline 0.05 & 12.8 & 10.8 \\
\hline 0.1 & 52.5 & 50.1 \\
\hline 0.2 & 30.9 & 33.2 \\
\hline 0.3 & 17.3 & 21.3 \\
\hline 0.4 & 15.7 & 12.7 \\
\hline 0.5 & 13.89 & 9.6 \\
\hline
\end{tabular}

\subsection{Effect of PVA concentration on the yield and embedding ratio of microcapsules}

The microcapsules are prepared with the stirring speed $1000 \mathrm{r} / \mathrm{min}$ at $60{ }^{\circ} \mathrm{C}$ with different concentrations of PVA aqueous solution. Effect of PVA concentration on the yield and embedding ratio of microcapsules is discussed and the results are shown in Table 3 and Fig. 3.

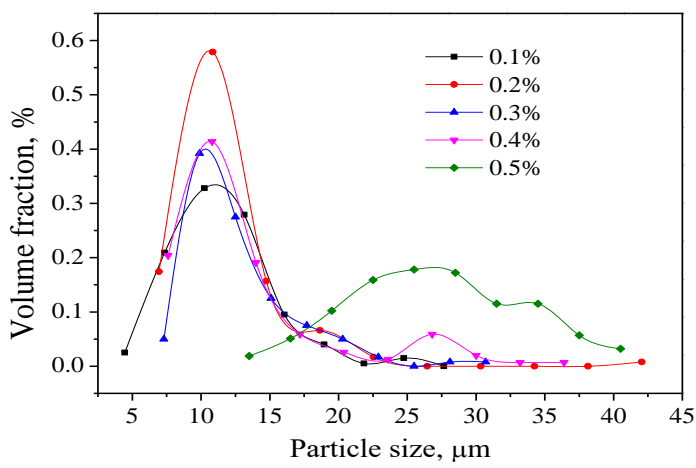

Fig. 3. Effect of PVA concentration on the distribution of the microcapsules size

As can be seen from Table 3, with the increasing mass concentration of PVA, the yield and embedding of the microcapsules increase firstly and then decrease. These two values of the microcapsule prepared with $0.1 \mathrm{wt} . \%$ PVA achieve the highest $52.5 \%$ and $50.1 \%$ respectively, which is comparable to that reported in other systems (55 wt.\%) [12]. It is because when the amount of PVA is lower, the dispersion effect of the system is worse. The viscosity of liquid system and the resistance of microspheres expansion increase with the increase of PVA content. So the particle size distribution showed in Fig. 3 is more uniform and the PUF aggregate quantity reduces gradually when PVA content increased. It also benefits the rise of yield. But when the concentration of PVA is more than $0.1 \mathrm{wt} . \%$, the microcapsule size distribution turn wider and the yield decreases. That's because more PVA will increase the thickness of the liquid film formed on the surface of vinyl silicone oil droplets. It will lead to the difficulty of migrating PUF molecular chain to the oilwater interface, which can prevent PUF depositing on the surface of the droplet and cause the yield of microcapsule reduced. When the concentration of PVA is more than $0.4 \mathrm{wt} . \%$, the microcapsules appear aggregation to some extent and the particle sizes increase accordingly. So the particles size of microcapsules with $0.5 \mathrm{wt} \%$ PVA concentration is much bigger than that of others, as can been seen from Fig. 3 .

\subsection{The morphology of microcapsules observed with PM and SEM}

The morphologies of PUF/vinyl silicone oil microcapsules are detected by PM and SEM and are shown in Fig.4. It is convinced that almost all the spherical microcapsules have smooth and dense surface, good dispersity, weak agglomeration as well as uniform distribution. The uniform particle size is within $5-20 \mu \mathrm{m}$ mostly. There are some PUF sediments attached to the surface of the particles as can be seen from the images. 


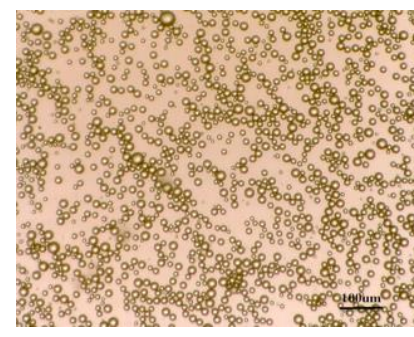

a

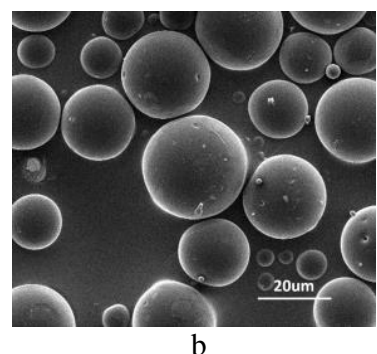

b
Fig. 4. a-PM image; b-SEM image of PUF/vinyl silicone oil microcapsules

\section{CONCLUSIONS}

In this study, PUF (as wall materials) and vinyl silicone (as core material) are used to prepare self-healing microcapsules and the optimal process parameters are found. When the reaction temperature is $60^{\circ} \mathrm{C}$, the stirring speed is $1000 \mathrm{r} / \mathrm{min}$ and PVA concentration is $0.1 \mathrm{wt} . \%$. The yield and embedding ratio of the microcapsules prepared reach $52.5 \%$ and $50.1 \%$, respectively. The microcapsules show smooth surface and good dispersion prepared under these process parameters. Particle size analysis results show that microcapsules have narrow particle size distribution and the average particle size is $13 \mu \mathrm{m}$.

\section{Acknowledgments}

The authors are grateful to the Xi'an Technological University Principal Fund (No.XAGDXJJ1409), Scientific Research Program Funded by Shaanxi Provincial Education Department (No.15JK1352) and Opening Fund of Shaanxi Key Laboratory of Photoelectric Functional Material and Devices (No.ZSKJ201418) for financial support.

\section{REFERENCES}

1. Neubauer, M.P., Poehlmann, M., Fery, A. Microcapsule Mechanics: From Stability to Function Advances in Colloid and Interface Science 207 2014: pp. 65-80.

2. Bari, M.F., Hossain, M.S., Mujtaba, I.M. Simultaneous Extraction and Separation of $\mathrm{Cu}(\mathrm{II}), \mathrm{Zn}(\mathrm{II}), \mathrm{Fe}(\mathrm{III})$ and $\mathrm{Ni}(\mathrm{II})$ by Polystyrene Microcapsules Coated with Cyanex 272 Hydrometallurgy 95 (3-4) 2009: pp. 308-315.
3. Yilmaz, A., Arslan, G., Tor, A. Selectively Facilitated Transport of Zn(II) Through a Novel Polymer Inclusion Membrane Containing Cyanex 272 as a Carrier Reagent Desalination $277(1-3)$ 2011: pp. 301-307.

4. Szabó, T., Telegdi, J., Nyikos,L. Linseed Oil-filled Microcapsules Containing Drier and Corrosion InhibitorTheir Effects on Self-healing Capability of Paints Progress in Organic Coatings 84 2015: pp. 136-142.

5. Huang, M.X., Yang, J.L. Salt Spray and EIS Studies on HDI Microcapsule-based Self-healing Anticorrosive Coatings Progress in Organic Coatings 77 (1) 2014: pp. $168-175$. http://dx.doi.org/10.1016/j.porgcoat.2013.09.002

6. Yang, Z.J., Ding, L., Wu, P. Fabrication of RDX, HMX and CL-20 Based Microcapsules via in situ Polymerization of Melamine-formaldehyde Resins with Reduced Sensitivity Chemical Engineering Journal 268 (15) 2015: pp. 60-66.

7. Salaün, F., Devaux, E., Bourbigot, S. Influence of Process Parameters on Microcapsules Loaded with n-hexadecane Prepared by in Situ Polymerization Chemical Engineering Journal 155 (1-2) 2009: pp. 457-465.

8. Ma, A.J., Zhang, Q.Y., Zhang, H.P., Liu, Y., Feng, X. Preparation of Self-Healing Coating Containing Vinyl Silicone Oil Microcapsules Paint \& Coatings Industry 43 (11) 2013: pp. 6-9.

9. Rule, J.D., Sottos, N.R., White, S.R. Effect of Microcapsule Size on the Performance of Self-healing Polymers Polymer 48 (12) 2007: pp. 3520-3529.

10. Keller, M.K., White, S.R., Sottos, N.R. A Self-healing Poly(dimethyl siloxane) Elastomer Advanced Functional Materials 17 (14) 2007: pp. 2399-2404. http://dx.doi.org/10.1002/adfm.200700086

11. Caruso, M.M., Delafuente, D.A., Ho, V., Moore, J.S., Sottos, N.R., White, S.R. Solvent-promoted Self-healing Epoxy Materials Macromolecules 40 (25) 2007: pp. $8830-8832$.

12. Fan, C.J., Zhou, X.D. Influence of Operating Conditions on the Surface Morphology of Microcapsules Prepared by in Situ Polymerization Colloids and Surfaces A: Physicochemical and Engineering Aspects $363(1-3)$ 2010: pp. 49-55.

13. McIlroy, D.A., Blaiszik, B.J., Caruso, M.M., White, S.R., Moore, J.S., Sottos, N.R. Microencapsulation of a Reactive Liquid-Phase Amine for Self-Healing Epoxy Composites Macromolecules 43 (4) 2010: pp. 1855-1859. 